\title{
ACCURACY ESTIMATION OF ASTRONOMICAL CONSTANTS FROM LONG BASELINE INTERFEROMETER OBSERVATIONS*
}

\author{
H. G. WALTER
}

European Space Operations Centre of ESRO, Darmstadt, F.R. Germany

\begin{abstract}
Inherent in Very Long Baseline Interferometry (VLBI) is the potentiality of determining relative angular positions of radio sources to an accuracy which is superior to present methods by at least one order of magnitude. For VLBI observations such as time delay, fringe frequency and fringe phase the sensitivity to the uncertainties of astronomical constants and geophysical parameters, i.e. luni-solar precession, nutation constant, rate of change of obliquity, polar motion and Earth rotation, is investigated.

The partial derivatives of the observations with respect to these unknowns form the core of the estimation process and give evidence of the influence of the source position and the baseline geometry on their magnitudes. By means of a variancecovariance analysis the standard deviations and the cross correlation coefficients of the unknowns are derived. It is inferred from them that VLBI observations are not only sensitive to variations in the astronomical constants and geophysical parameters, but that the effects caused by these variations are also measurable and separable, if the observations are extended over a sufficiently long interval of time, and the baselines and source positions are chosen appropriately.
\end{abstract}

\section{DISCUSSION}

Fricke: Can you say how the accuracy of the determinations changes, if the number of unknowns is decreased, in particular, if you don't include as an unknown the rate of change of the obliquity?

Walter: Since the unknowns are usually correlated, an apparent improvement of accuracy is obtained if a smaller number of unknowns is processed. Moreover, the large time spacing of observations over 10 years or more would be avoidable. In order to arrive at a meaningful error estimation, however, the number of unknowns should not be unnecessarily diminished.

* The full text of this paper is being published as Bull. GRGS, No. 10 of the Groupe Recherche Géodesié Spatiale, Observatoire de Paris, Meudon, France. 